\title{
Ablation d'un bloc de glace transparente par un jet d'eau chaude - Application à la sûreté des RNR-Na
}

\section{Ablation of ice bloc by a hot water jet - Application to SFR safety}

\author{
Alexandre Lecoanet ${ }^{1,2,}$, Nicolas Rimbert $^{2}$, Michel Gradeck ${ }^{2}$, Frédéric Payot ${ }^{1}$ \\ ${ }^{1}$ CEA, DEN, Cadarache, DTN, SMTA, LEAG, 13108 St Paul-lès-Durance, France \\ ${ }^{2}$ Université de Lorraine, CNRS, LEMTA, F-54000, Nancy, France \\ * (auteur correspondant : alexandre.lecoanet@univ-lorraine.fr)
}

\begin{abstract}
RÉSUMÉ. L'ablation d'un bloc de glace par un jet d'eau chaude est étudiée dans le but d'obtenir une compréhension physique du phénomène pouvant in fine être appliqué au dimensionnement du récupérateur de corium d'un RNR-Na. Le système étudié met en œuvre un jet d'eau et un bloc de glace transparente. Le suivi en temps réel de l'ablation est visualisé par une caméra rapide. Les résultats présentés montrent la succession des régimes rencontrés. Un nouveau régime d'ablation est mis en évidence. L'évolution de la vitesse d'ablation en fonction du temps et de la vitesse du jet est analysée.

ABSTRACT. Ablation of an ice bloc by a hot water jet is studied to understand the physical phenomena involved in the process, the final goal is to design SFR's core-catcher. The system studied is comprised of a water jet and a transparent ice block. Ablation process is followed by use of high-speed camera and back lighting. First results are presented. A new ablation regime is reported. The evolution of ablation speed with time and jet speed is analyzed.

MOTS-CLÉS. Ablation, Fonte, Jet liquide, Sûreté nucléaire, Film liquide, Interaction fluide-structure, Changement de phase.

KEYWORDS. Ablation, Melting, Liquid jet, Nuclear safety, Liquid film, Fluid-structure interaction, Phase change.
\end{abstract}

NOMENCLATURE.

\begin{tabular}{|c|c|c|c|c|c|}
\hline$C_{p}$ & $J \cdot \mathrm{kg}^{-1} \cdot \mathrm{K}^{-1}$ & Capacité thermique massique & \multicolumn{3}{|c|}{ SYMBOLES GRECS } \\
\hline$D$ & $m$ & Diamètre & $\mu$ & Pa.s & Viscosité dynamique \\
\hline$g$ & $m \cdot s^{-2}$ & Accélération de la pesanteur & $\rho$ & $\mathrm{kg} \cdot \mathrm{m}^{-3}$ & Masse volumique \\
\hline$h$ & $W \cdot m^{-2} \cdot K^{-1}$ & Coefficient d'échange convectif & \multicolumn{3}{|c|}{ INDICES ET EXPOSANTS } \\
\hline$H$ & $m$ & Hauteur de chute & 0 & \multicolumn{2}{|c|}{ Sortie de buse ou initial } \\
\hline$k$ & $W \cdot m^{-1} \cdot K^{-1}$ & Conductivité thermique & 1 & \multicolumn{2}{|c|}{ Avant le début du «pool-effect » } \\
\hline$L$ & $J \cdot \mathrm{kg}^{-1}$ & Chaleur latent de fusion & 2 & \multicolumn{2}{|c|}{ Après le début du « pool-effect » } \\
\hline$q$ & $W \cdot m^{-2}$ & Densité de flux thermique & eff & \multicolumn{2}{|l|}{ Effectif } \\
\hline$N u$ & - & Nombre de Nusselt $h D / k$ & $\begin{array}{c}j \\
p e\end{array}$ & «pool-effect » & \\
\hline $\operatorname{Pr}$ & - & Nombre de Prandtl $\mu C_{p} / k$ & $\begin{array}{c}S \\
\text { ACRONYMES }\end{array}$ & \multicolumn{2}{|l|}{ Solide } \\
\hline$R e$ & - & Nombre de Reynolds $\rho V D / \mu$ & RNR-NA & $\begin{array}{l}\text { Réacteur à } \\
\text { Sodium }\end{array}$ & Neutrons Rapides refroidis au \\
\hline$V$ & $m \cdot s^{-1}$ & Vitesse & LEMTA & \multicolumn{2}{|c|}{$\begin{array}{l}\text { Laboratoire Energie \& Mécanique Théorique } \\
\text { et Appliquée }\end{array}$} \\
\hline
\end{tabular}




\section{Introduction}

La possibilité de formation d'un magma constitué d'un mélange multiphasique de combustible nucléaire et d'éléments de structures fondus (corium) dans le cœur d'un réacteur nucléaire à neutrons rapides refroidi au sodium (RNR-Na) est prise en compte dès les premières phases de dimensionnement [BER18]. L'un des enjeux consiste à extraire le corium du cœur aussi vite que possible pour éviter tout risque de re-criticité en augmentant les fuites neutroniques ainsi que pour améliorer son refroidissement ultérieur. Pour atteindre cet objectif, la solution proposée consiste à disposer des tubes de déchargement en cœur et débouchant dans le caloporteur en dessous de celuici et des structures de maintien présentes dans la cuve. Pour augmenter la surface d'échange avec le caloporteur et protéger la deuxième barrière de confinement ${ }^{1}$, un plateau récupérateur de corium est disposé sur le fond de cuve (Fig. 1). En situation accidentelle avec décharge du corium, ce dernier dont la température est supérieure à $2000 \mathrm{~K}$ - elle dépend de sa composition - impactera le récupérateur sous forme de jet. Dans ce cas de figure, il est probable qu'une partie du matériau composant le récupérateur fonde. Il faut donc le dimensionner en conséquence.

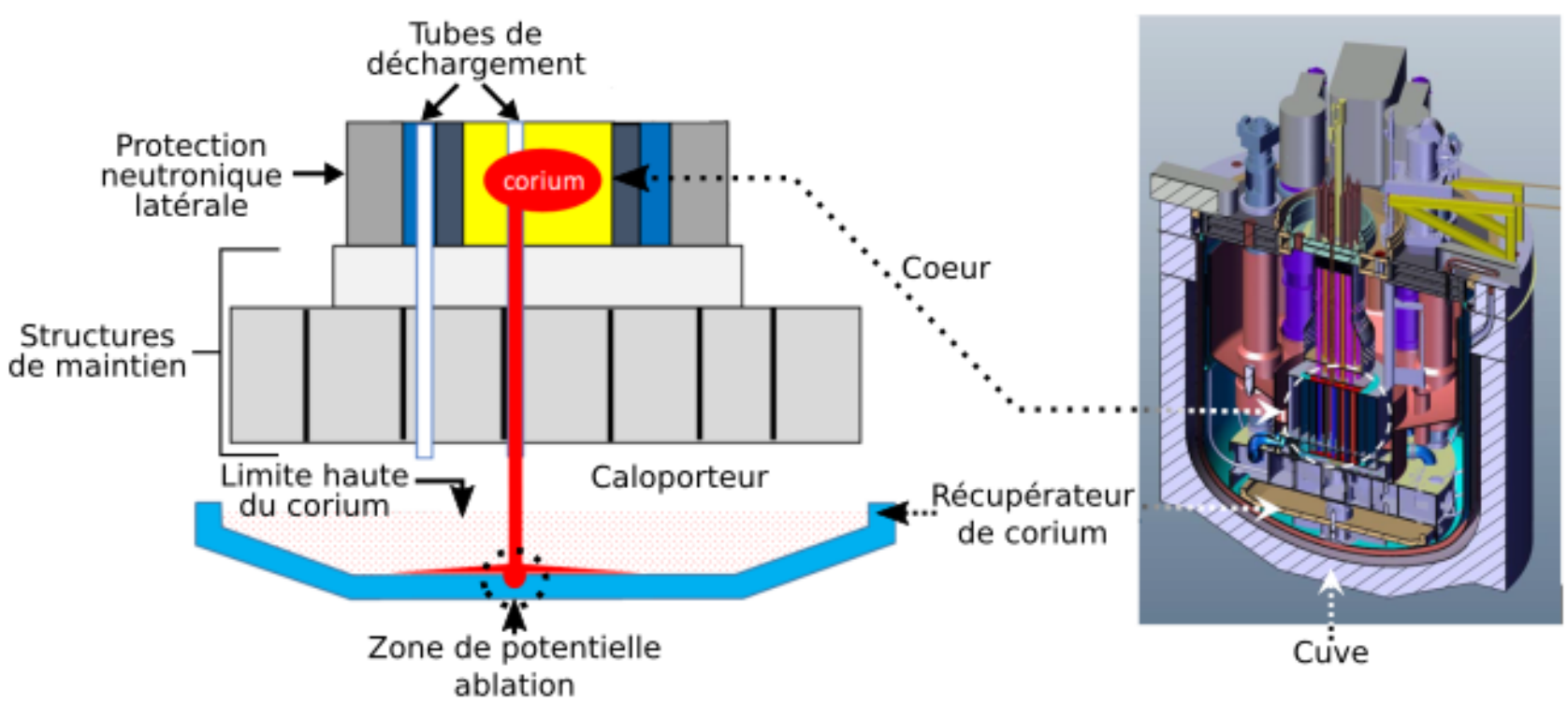

Figure 1. Schéma de principe du couple tubes de déchargement récupérateur de corium (gauche). Exemple de coupe de réacteur nucléaire de quatrième génération incluant des tubes de déchargement et un récupérateur en cuve (droite).

Une certaine fraction du corium devrait se fragmenter lors de sa pénétration dans le caloporteur, si ce dernier est toujours liquide. Néanmoins, on considère que l'élargissement du jet de corium et l'augmentation de la surface de contact entre ce dernier et le caloporteur, consecutifs à la fragmentation, sont de nature à réduire la densité de flux thermique appliquée sur le récupérateur. C'est donc l'impact d'un jet cohérent qui entraîne la plus grande densité de flux sur le récupérateur et donc un percement par changement de phase (ablation) plus rapide. Les compositions relatives du corium et du récupérateur peuvent aussi jouer un rôle important sur la vitesse d'ablation. Les plus grandes vitesses d'ablation sont atteintes lorsque le corium et le récupérateur sont de même nature [LEC19].

Des études ont déjà été menées dans le contexte de la sûreté nucléaire [POW84,SAI90,SAT91] ; néanmoins, seules de faibles épaisseurs de parois étaient considérées. De plus, la vitesse d'ablation a toujours été considérée comme constante lors de ces études. Elles ont cependant mis au jour un régime pendant lequel le film liquide engendré par la déflexion du jet s'effondre pour donner

\footnotetext{
${ }^{1}$ C'est-à-dire la cuve du réacteur.
} 
naissance à une piscine de liquide. Durant ce régime, nommé «pool-effect» [SAI90], la vitesse d'ablation diminue et il n'a pas été caractérisé plus avant. Aussi très peu d'informations sont disponibles sur la géométrie globale de la cavité lors de l'ablation, le rythme d'ablation et les coefficients d'échanges associés.

Pour mieux comprendre le processus d'ablation dans son ensemble et comprendre l'apparition du «pool-effect », il faut avoir accès à la géométrie de la cavité en temps réel. Le couple eau/glace transparente a donc été choisi pour simuler l'ablation d'un bloc solide soumis à l'impact d'un jet liquide de même nature. Le principal avantage est de pouvoir suivre le déroulement de l'ablation en temps réel. Pour mettre en œuvre ce système, le dispositif HAnSoLO (Hot AblatioN of a SOlid by a Liquid - Observation) a été conçu au LEMTA. Les données obtenues et leur analyse permettront in fine de tester la robustesse des futurs codes de calcul développés au CEA qui serviront aux études de sûreté des RNR-Na.

\section{Dispositif expérimental}

Le but du dispositif HAnSoLO est de reproduire au maximum les conditions d'ablation probables lors de l'impact d'un jet de corium sur un récupérateur métallique tout en permettant une métrologie fine et une répétition d'expériences rendant possible la compréhension des mécanismes à l'œuvre. Les paramètres étudiés sont la vitesse du jet, sa température et son diamètre. Un schéma légendé du dispositif HAnSoLO est reproduit sur la Fig. 2. En plus de la caméra rapide enregistrant la formation de la cavité de côté, une caméra standard et une caméra thermique sont pointées vers la zone d'impact $^{2}$.

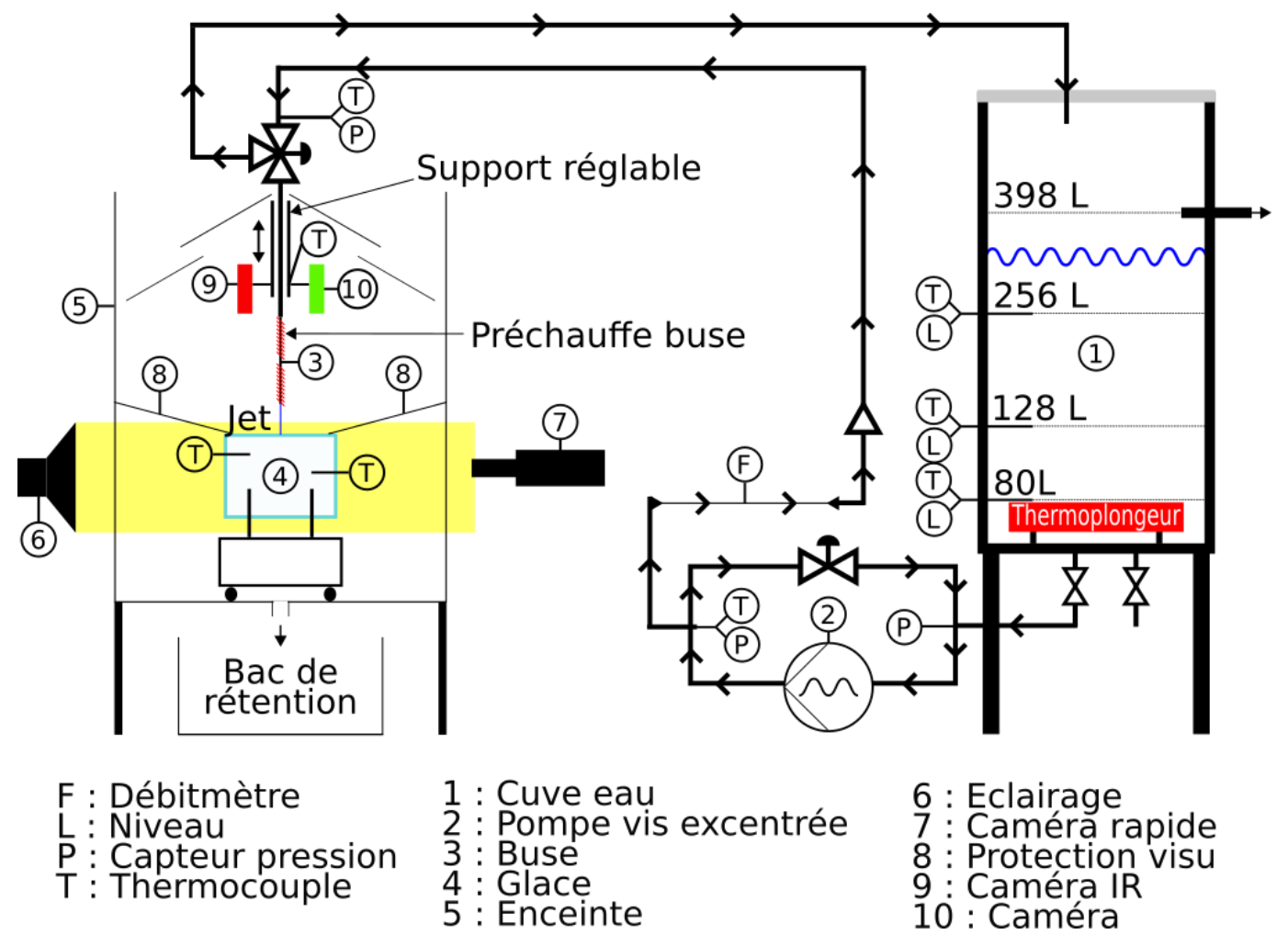

Figure 2. Schéma du montage expérimental HAnSoLO mis au point au LEMTA.

En plus d'être transparent, le système eau / glace ne forme pas de croûte une fois l'ablation démarrée, ce qui est représentatif du système corium métallique / récupérateur de corium métallique. Dans le cas de l'impact d'un jet de corium, on s'attend à ce que la hauteur de chute soit supérieure à

\footnotetext{
${ }^{2}$ Elles permettent notamment de repérer d'éventuelles perturbations induites par les systèmes de protection ou refroidissement du liquide sur son trajet vers la sortie de la buse.
} 
six fois le diamètre [TON03] du jet et soit donc suffisante pour que le profil de vitesse dans le jet soit uniforme. Le profil de vitesse dans le jet influant les transferts thermiques à l'impact [LIE06], la hauteur de chute du jet est donc réglable dans l'expérience HAnSoLO. Le jet produit dans l'installation expérimentale se développe dans un milieu gazeux. Dans le cas d'un jet de corium se développant dans un plenum de sodium liquide, une certaine fragmentation du jet est inévitable, mais ce phénomène sort du cadre de notre étude. Du fait de la température du corium (> $2000 \mathrm{~K})$, il est très probable qu'une couche, voire une poche, de gaz se forme rapidement autour du jet.

Le nombre de Prandtl dicte notamment l'évolution relative [LIE06] des couches limites thermiques et hydrodynamiques se développant à partir du point d'impact du jet ; c'est donc un paramètre important pouvant potentiellement avoir une influence sur la forme de la cavité. L'influence de ce paramètre est étudiée en faisant varier la température du jet (Tab. 1). Néanmoins, le nombre de Prandtl de l'eau est toujours supérieur à l'unité, tandis qu'il est inférieur à un pour les métaux liquides. Malgré cette différence les formes de cavités obtenues post-mortem par Sato et al. [SAT91] semblent en accord avec les formes que nous avons obtenues (Fig. 3). Il faut préciser que les images obtenues dans HAnSoLO sont des projections de la forme de la cavité. Donc d'éventuelles bosses axisymétriques, telles que celles visibles sur la coupe de Sato et al. [SAT91], apparaîtraient noires comme la cavité remplie d'air.

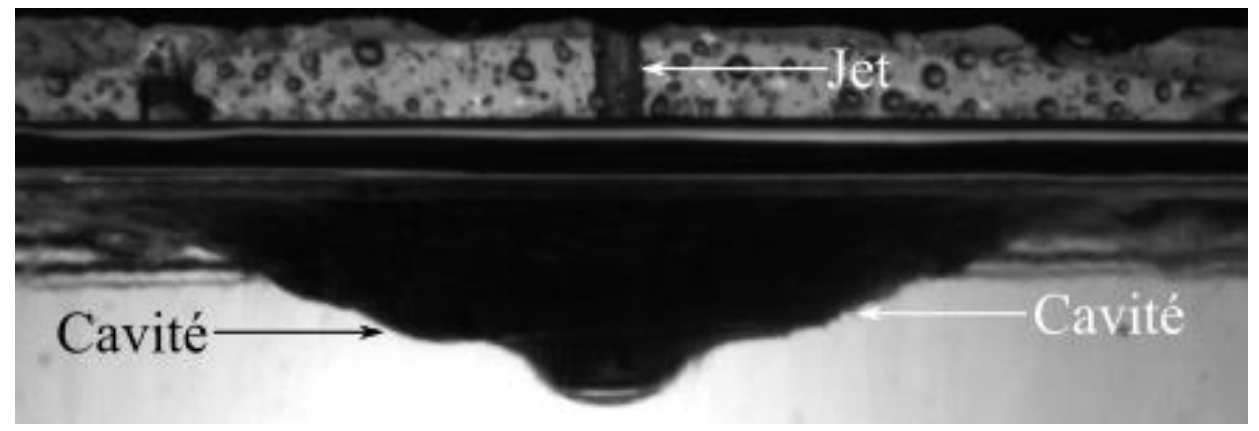

Figure 3. Test eau/glace transparente avec un jet de $5,9 \mathrm{~mm}$ à une température de $50{ }^{\circ} \mathrm{C}$ et une vitesse de jet de 4,5 m/s (Re = 47500 et $\mathrm{Pr}=3,6)$. La forme de la cavité est très proche de la géométrie révélée par la coupe post-mortem rapportée par Sato et al. [SAT91] (Fig. 6d dans l'article de Sato et al.).

L'étendue des paramètres possibles avec HAnSoLO est reportée dans le tableau 1. Dans le cas de l'impact d'un jet de corium, les températures du jet seraient supérieures à $2000 \mathrm{~K}$, sa vitesse estimée est d'environ $10 \mathrm{~m} / \mathrm{s}$ et son diamètre à $8 \mathrm{~cm}$ au maximum. Le nombre de Reynolds à l'impact serait ainsi de l'ordre de $10^{6}$. HAnSoLO se rapproche du cas réel en terme de nombre de Reynolds. La température initiale du bloc impacté a pour effet principal d'induire un retard au démarrage de l'ablation. L'impact d'un jet d'eau chaude sur un bloc de glace peut produire un éclatement du bloc. C'est un mécanisme en dehors du périmètre de l'étude. Donc la température de la glace est mesurée durant les expériences et l'expérience n'est conduite que si la température du bloc est comprise entre $-2{ }^{\circ} \mathrm{C}$ et $0{ }^{\circ} \mathrm{C}$.

\begin{tabular}{|c|c|c|}
\hline & Minimum & Maximum \\
\hline Vitesse du jet & $1,0 \mathrm{~m} / \mathrm{s}$ & $10,0 \mathrm{~m} / \mathrm{s}$ \\
\hline Température du jet & $20{ }^{\circ} \mathrm{C}$ & $80^{\circ} \mathrm{C}$ \\
\hline Diamètre de la buse & $3,0 \mathrm{~mm}$ & $10,0 \mathrm{~mm}$ \\
\hline $\operatorname{Re}$ & 4000 & 270000 \\
\hline $\operatorname{Pr}$ & 2,5 & 7,0 \\
\hline
\end{tabular}

Tableau 1. Tableau des conditions possibles avec l'installation HAnSoLO. 


\section{Résultats expérimentaux et analyse}

Le tableau 2 résume l'ensemble des données obtenues dans le cas d'un jet à $50{ }^{\circ} \mathrm{C}$. La figure 4 permet de comparer le déroulement de l'ablation dans deux cas. Le jet commence par impacter la surface et former un film liquide sur le solide. L'ablation commence puis le film se décolle pour former une nappe liquide qui se rapproche ensuite de la surface avant de la rejoindre. Dès le début de l'ablation, la cavité prend une forme tronconique autour de la zone d'impact. À cette forme succède un plateau qui est d'autant plus proche de la zone d'impact que la vitesse du jet est grande. Nous supposons que ce plateau (Fig. 4) peut être dû à une zone de recirculation induite par l'apparition de la turbulence dans le film. Des expériences complémentaires sont en cours de réalisation pour tenter de déterminer la validité de cette hypothèse. Après un certain temps (reporté en Tab. 2), le film se déstabilise et s'effondre dans la cavité. Le jet impacte donc une cavité remplie de liquide, c'est le «pool-effect ». La profondeur de cavité pour laquelle le «pool-effect » apparaît varie entre six et douze fois le diamètre du jet à l'impact, ce n'est donc pas un paramètre suffisant pour prédire l'apparition du «pool-effect». Après l'apparition du «pool-effect», la forme de la cavité change, traduisant un changement des transferts thermiques et massiques.

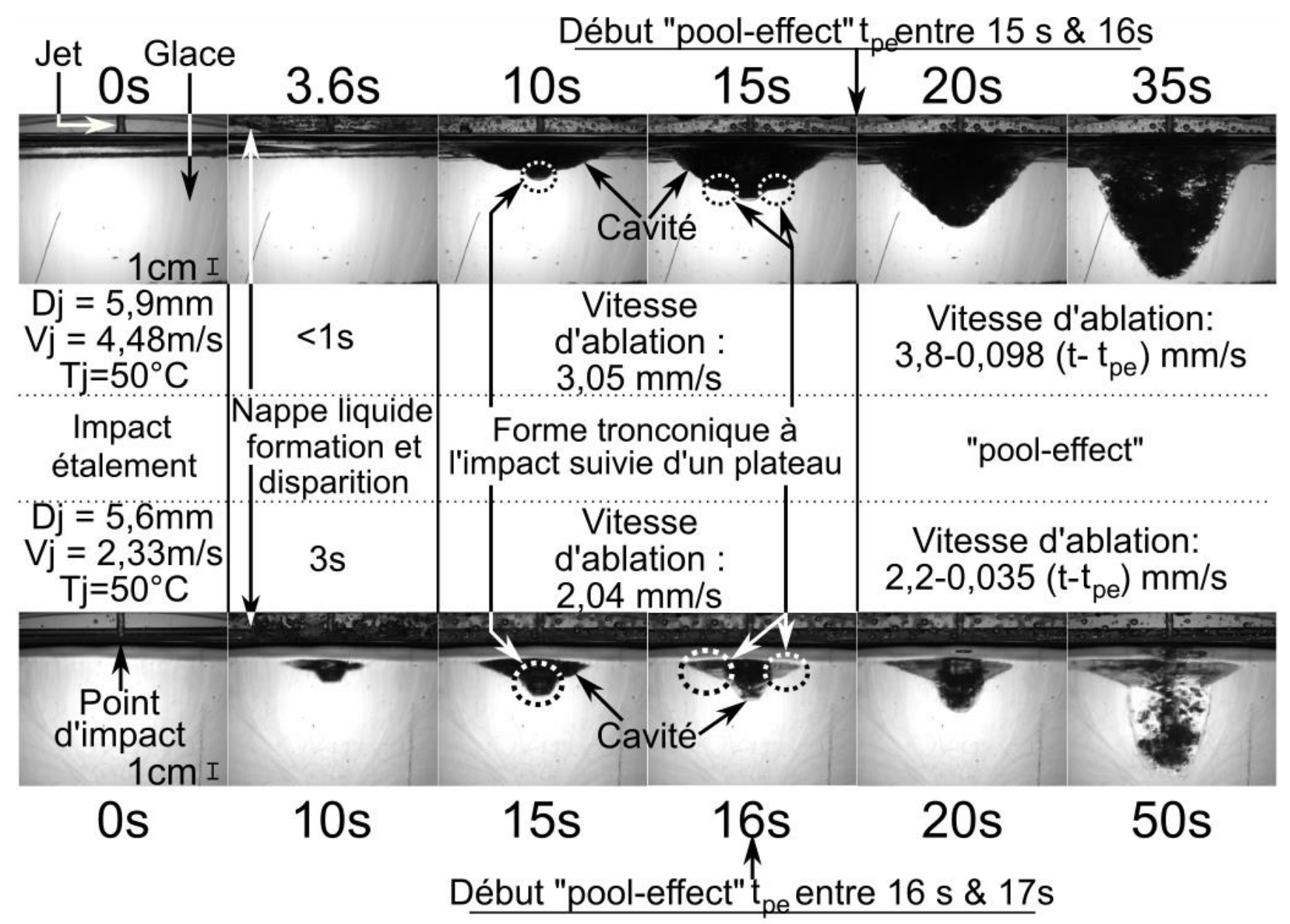

Figure 4. Comparatif de l'évolution de la forme de la cavité au cours du processus d'ablation pour deux vitesses de jet différentes.

La vitesse moyenne en sortie de buse du jet est déterminée, connaissant le diamètre de la buse, grâce à un débitmètre électromagnétique. Pour obtenir la vitesse à l'impact du jet, une correction prenant en compte l'accélération de la gravité est appliquée. L'accélération induit une diminution du 
diamètre du jet obtenue par conservation du débit (Eq. (1)). Ces corrections sont surtout importantes pour les vitesses inférieures à $5 \mathrm{~m} / \mathrm{s}$, au-dessus la correction est inférieure à $2 \%{ }^{3}$.

$$
\mathrm{V}_{\mathrm{j}}=\sqrt{V_{0, j}^{2}+2 g H} \quad ; \quad D_{j}=D_{j, 0}\left(1+\frac{2 g H}{V_{0, j}^{2}}\right)^{-1 / 4}
$$

La forme de la cavité est obtenue par traitement d'images. On en déduit la vitesse du front d'ablation dans l'axe du jet. La position de l'interface au cours du temps (Fig. 5) est estimée avant et après le «pool-effect». La dérivée des résultats de régression donne la vitesse avant et après le début du «pool-effect». Les résultats sont consignés dans le tableau 2. On peut constater sur les données de régression (Tab. 2 et Fig. 5) que, contrairement à ce qui a été observé sur des tests effectués avec de plus petits diamètres [LEC19], la réduction de vitesse d'ablation ne survient pas dès le début du «pool-effect», mais qu'au contraire la vitesse d'ablation peut légèrement augmenter ${ }^{4}$. Ce régime transitoire n'a, à notre connaissance, jamais été décrit auparavant.

L'utilisation de la condition de saut sur la densité de flux thermique (2), permet de relier la vitesse d'ablation au coefficient d'échange convectif par le biais de l'équation (4), en postulant (3) et en négligeant $\mathrm{q}_{\mathrm{s}}$, ce qui est équivalent à considérer que la glace à est $0{ }^{\circ} \mathrm{C}$ lors de l'expérience.

$$
\begin{aligned}
& q_{j}-q_{s}=\rho_{s} V_{f} L \\
& q_{j}=h_{e f f}\left(T_{0, j}-T_{f, s}\right) \\
& \rho_{s} V_{f} L=h_{e f f}\left(T_{0, j}-T_{f, s}\right) ; N u_{e f f}=\frac{h_{e f f} D_{j}}{k_{j}}
\end{aligned}
$$

En utilisant cette méthode, Sato et al. [SAT91] ont déterminé la corrélation reportée ci-dessous (Eq. (5)). Par un traitement similaire, une autre corrélation (Eq. (6)) a été déterminée [LEC19] pour le système eau/glace avec un diamètre de jet de $1 \mathrm{~mm}$. Les nombres de Nusselt obtenus par ces corrélations sont comparés à ceux déterminés expérimentalement (Eq. (4)). Ces nombres de Nusselt ont été obtenus pour des vitesses d'ablation constantes ${ }^{5}$. On remarque que la corrélation de Sato et al. [SAT91] surestime les transferts thermiques, ce qui peut être dû à la différence entre les nombres de Prandtl (métaux liquides contre de l'eau dans notre cas). Il en est de même pour la corrélation (6) obtenue précédemment au LEMTA [LEC19], ce qui peut indiquer une forte dépendance du nombre de Nusselt au diamètre du jet.

$$
\begin{aligned}
& N u_{e f f}=0,0152 R e_{j}^{0,92} P r_{j}^{0,8} ; P r_{j} \in[0,0095-0,20] ; \quad R e_{j} \in\left[4,1.10^{4}-4,9.10^{5}\right] \\
& N u_{e f f}=0,121 R e_{j}^{0,6} P r_{j}^{0,8} ; P r_{j} \in[2,55-5,42] ; \quad R e_{j} \in\left[3,8.10^{3}-1,2.10^{4}\right]
\end{aligned}
$$

\footnotetext{
${ }^{3}$ En considérant une hauteur de chute de dix fois le diamètre avec le plus gros diamètre $(1 \mathrm{~cm})$ utilisable dans l'installation HAnSoLO.

${ }^{4}$ C'est notamment ce que traduit la différence entre la valeur avant « pool-effect » de la vitesse d'ablation et la partie constante de l'équation donnant l'évolution de la vitesse d'ablation au cours du temps pour un même test.

${ }^{5}$ Avant le début du « pool-effect ».
} 


\begin{tabular}{|c|c|c|c|c|c|c|c|c|c|c|}
\hline \multicolumn{5}{|c|}{ Paramètres d'entrée } & \multicolumn{6}{|c|}{ Paramètres de sortie } \\
\hline$V_{j}$ & \multirow{2}{*}{$\begin{array}{c}D_{j} \\
\text { m }\end{array}$} & \multirow{2}{*}{$\begin{array}{l}T_{j} \\
{ }^{\circ} \mathrm{C}\end{array}$} & \multirow[t]{2}{*}{$R e_{j}$} & \multirow[t]{2}{*}{$\operatorname{Pr}_{j}$} & \multirow{2}{*}{$\begin{array}{l}V_{f 1} \\
\mathrm{~m} / \mathrm{s}\end{array}$} & \multirow{2}{*}{$\begin{array}{c}\boldsymbol{t}_{p e} \\
\mathrm{~s}\end{array}$} & \multirow{2}{*}{$\begin{array}{l}V_{f 2} \\
\mathrm{~m} / \mathrm{s}\end{array}$} & \multicolumn{3}{|c|}{$N u_{e f f}$} \\
\hline $\mathrm{m} / \mathrm{s}$ & & & & & & & & Exp. & $\begin{array}{l}\text { [SAT91] } \\
\text { Eq. (5) }\end{array}$ & $\begin{array}{c}\text { [LEC19] } \\
\text { Eq. (6) }\end{array}$ \\
\hline 1,22 & $4,0.10^{-3}$ & & 8786 & & $1,67.10^{-3}$ & 16,8 & $\begin{array}{c}1,51.10^{-3} \\
-2,21 \cdot 10^{-5}\left(t-t_{\mathrm{pe}}\right) \\
\end{array}$ & 63,5 & 180 & 78,4 \\
\hline 2,33 & $5,6.10^{-3}$ & & 22685 & & $2,04.10^{-3}$ & 16,2 & $\begin{array}{c}2,16.10^{-3} \\
-3,54 \cdot 10^{-5}\left(t-t_{p e}\right) \\
\end{array}$ & 109 & 445 & 141 \\
\hline 4,43 & $5,9.10^{-3}$ & 50 & 47491 & 3,6 & $3,05.10^{-3}$ & 15,2 & $\begin{array}{c}3,8 \cdot 10^{-3} \\
-9,76 \cdot 10^{-5}\left(t-t_{p e}\right) \\
\end{array}$ & 171 & 850 & 216 \\
\hline 7,57 & $6010^{-3}$ & & 81772 & & $3,88.10^{-3}$ & 15,4 & $\begin{array}{c}4,63 \cdot 10^{-3} \\
-10,3 \cdot 10^{-5}\left(t-t_{p e}\right) \\
\end{array}$ & 221 & 1401 & 299 \\
\hline 10,0 & $0,0.10$ & & 108022 & & $4,96.10^{-3}$ & 14,9 & $\begin{array}{c}6,90 \cdot 10^{-3} \\
-22,5 \cdot 10^{-5}\left(t-t_{\mathrm{pe}}\right) \\
\end{array}$ & 283 & 1810 & 353 \\
\hline
\end{tabular}

Tableau 2. Récapitulatif des tests effectués avec un diamètre de buse de $6 \mathrm{~mm}$ et une température de 50 ${ }^{\circ} \mathrm{C}$. Le calcul des nombres de Nusselt a été effectué pour la partie avant l'apparition du " pool-effect » pour laquelle la vitesse de fonte est constante.

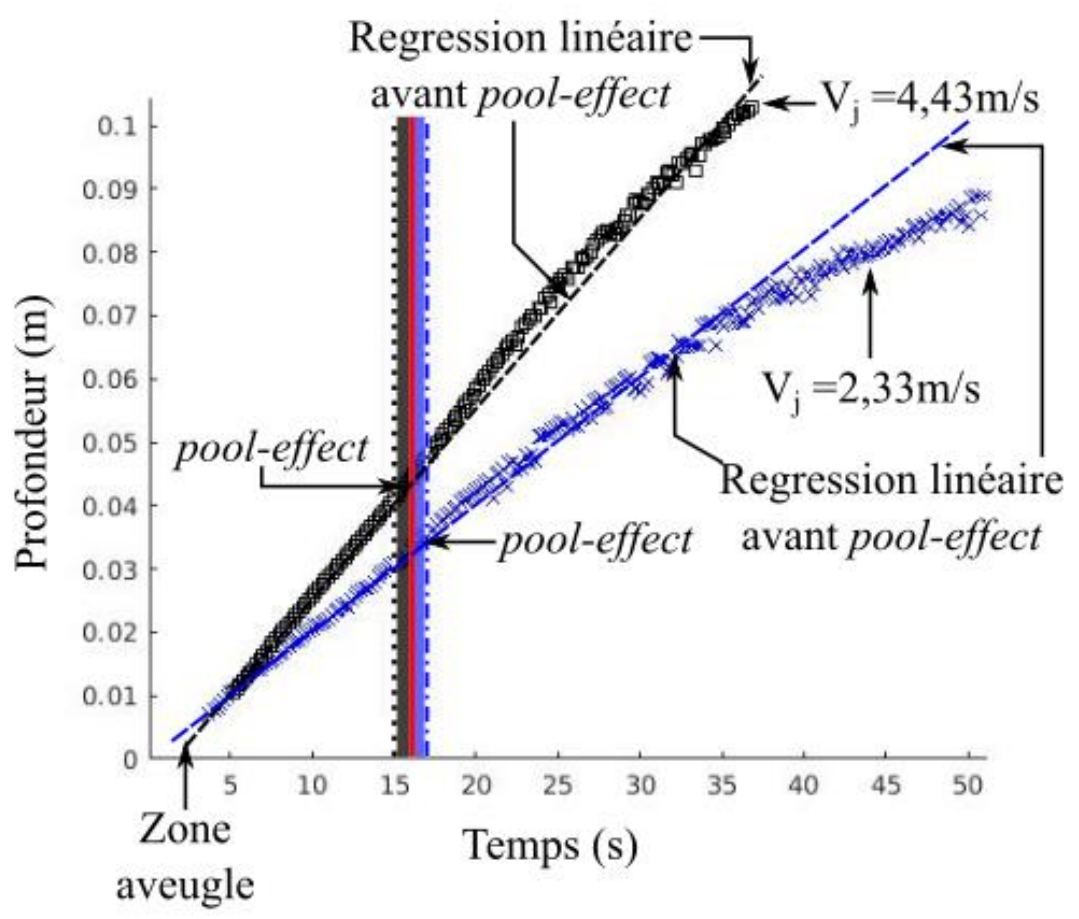

Figure 5. Evolution de la profondeur d'ablation en fonction du temps pour les deux cas présentés sur la Fig.

4. Les carrés noirs correspondent au cas où $V_{j}=4,43 \mathrm{~m} / \mathrm{s}$. Le «pool-effect » apparaît entre la ligne en pointillés noirs et la ligne continue rouge. Les croix bleues correspondent au cas $V_{j}=2,33 \mathrm{~m} / \mathrm{s}$, Le "pool-effect » apparaît entre la ligne continue rouge et la ligne discontinue bleue. Un agrandissement est effectué sur la zone suivant le «pool-effect ». 


\section{Conclusion}

Ces essais montrent la représentativité phénoménologique du dispositif HAnSoLO construit et opéré par le LEMTA. Le déroulé de la séquence d'impact est similaire à ce qui a été obtenu par le passé lors de tests acier/acier [SAT91]. La forme de la cavité est aussi conforme à ce qui a été observé post-mortem lors de ces mêmes expériences. La constance de la vitesse d'ablation a de nouveau été montrée. L'existence d'un régime de transition au début du «pool-effect» a été découverte. Une étude paramétrique est en cours de réalisation au LEMTA pour quantifier l'influence de la température, de la vitesse et du diamètre du jet sur la cinétique d'ablation et le régime de transition et obtenir une relation de type (6) en ayant fait varier le diamètre du jet. L'enjeu, au centre des développements futurs de cette étude, est l'obtention de lois ou de corrélations permettant de prédire la forme de la cavité. Ces données permettront de mieux dimensionner les zones du récupérateur de corium disposées en dessous des sorties des tubes de déchargement.

\section{Remerciements}

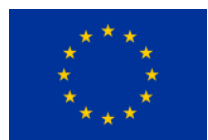

The research leading to these results has received funding from the Euratom research and training program 2014-2018 under grant agreement No 754501.

\section{Bibliographie}

[BER18] Bertrand F., Marie N., Bachrata A., Brun-Magaud V., Droin J. B., Manchon X., Herbreteau K., Farges B., CARluec B., Poumerouly S., LEMASSON D., «Status of severe accident studies at the end of the conceptual design of ASTRID: Feedback on mitigation features », Nuclear Engineering and Design, Vol. 326, p. $55-64,2018$

[LEC19] Lecoanet A., Rimbert N., PAyot F., GradeCK M., «Etude de l'ablation d'une paroi solide par un jet liquide », 24ème Congrès Français de Mécanique, Brest, France, 26-30 Août, 2019

[LIE06] LIENHARD J. H., « Heat Transfer by Impingement of Circular Free-Surface Liquid Jets », 18th National \& 7th ISHMT-ASME Heat and Mass Transfer Conference, IIT Guwahati, Inde, 4-6 Janvier, 2006

[POW84] POWERS D. A., «Erosion of Steel Structures by High-Temperature Melts », Nuclear Science and Engineering, Vol. 88, n 3, p. 357-366, 1984

[SAI90] SAito M., SATO K., FURUTANi A., ISOZAKi M., IMAHORI S., HATTORI Y., « Melting attack of solid plates by a high temperature liquid jet -- effect of crust formation », Nuclear Engineering and Design, Vol. 121, n 1, p. 11-23, 1990

[SAT91] Sato K., Furutani A., Saito M., IsOZaKi M., Suganuma K., Imahori S., « Melting attack of solid plates by a high-temperature liquid jet [II] -- Erosion behavior by a molten metal jet », Nuclear Engineering and Design, Vol. 132, n 2, p. 171-186, 1991

[TON03] Tong A. Y., « A Numerical Study on the Hydrodynamics and Heat Transfer of a Circular Liquid Jet Impinging Onto a Substrate », Numerical Heat Transfer, Part A: Applications, Vol. 44, n 1, p.99-105, 2003 